\title{
Insights into the Off-State Breakdown Mechanisms in Power GaN HEMTs
}

\author{
N. Zagni ${ }^{\mathrm{a},}{ }^{\dagger}$, F.M. Puglisi ${ }^{\mathrm{a}}$, P. Pavan ${ }^{\mathrm{a}}$, A. Chini ${ }^{\mathrm{a}}$, G. Verzellesi ${ }^{\mathrm{b}}$ \\ a DIEF, Univ. of Modena and Reggio Emilia, Modena, Italy \\ ${ }^{\mathrm{b}}$ DISMI and EN\&TECH, Univ. of Modena and Reggio Emilia, Reggio Emilia, Italy
}

\begin{abstract}
We analyse the off-state, three-terminal, lateral breakdown in AlGaN/GaN HEMTs for power switching applications by comparing two-dimensional numerical device simulations with experimental data from device structures with different gate-to-drain spacing and with either undoped or Carbon-doped GaN buffer layer. Our simulations reproduce the different breakdown-voltage dependence on the gate-drain-spacing exhibited by the two types of device and attribute the breakdown to: $i$ ) a combination of gate electron injection and source-drain punchthrough in the undoped HEMTs; and ii) avalanche generation triggered by gate electron injection in the C-doped HEMTs.
\end{abstract}

\section{Introduction}

In $\mathrm{AlGaN} / \mathrm{GaN}$ HEMTs for power switching applications, the three-terminal off-state breakdown voltage $\left(V_{\mathrm{BR}}\right)$ is typically extended up to the vertical breakdown limit by compensating the unintentional conductivity in the buffer through Carbon (C) doping and by increasing the lateral gate-to-drain spacing $\left(L_{\mathrm{GD}}\right)[1] . V_{\mathrm{BR}}$ is typically found to scale about linearly with $L_{\mathrm{GD}}$ with a $\Delta V_{\mathrm{BR}} / \Delta L_{\mathrm{GD}}$ slope that is smaller than the critical field for avalanche $\left(E_{\mathrm{CRIT}}=3.9 \mathrm{MV} / \mathrm{cm}\right.$ [2]). This is often considered to be an indication that avalanche generation should be ruled out as the $V_{\mathrm{BR}}$ limiting phenomenon. Doing so corresponds, however, to assuming a quite idealized, constant electric-field distribution throughout the access region between gate and drain. The latter is on the contrary two-dimensional and, above all, characterized by intense accumulation spots at the drain-end of the gate, under the field-plate end (if present), and at the drain contact border. Moreover, it is critically impacted by the intrinsic or doping-related traps in the buffer. For these reasons, numerical device simulations are probably the only means by which the role possibly played by avalanche generation in the off-state breakdown can be clarified.

Paper [1] by Bahat-Treidel et al. is one of the very few works in the open literature providing a systematic analysis of the $V_{\mathrm{BR}}$ vs $L_{\mathrm{GD}}$ dependence in $\mathrm{AlGaN} / \mathrm{GaN}$ HEMTs with and without $\mathrm{C}$ doping in

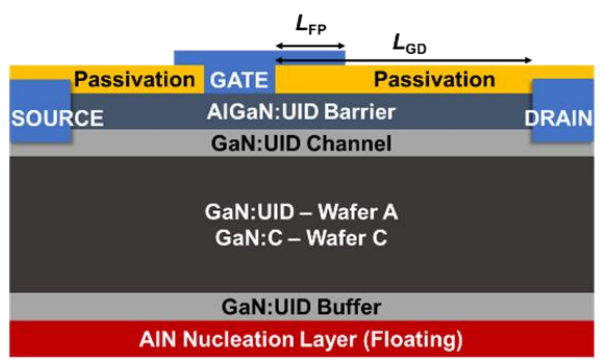

Fig. 1. Schematic cross-section of the GaN HEMT device without (Wafer $\mathrm{A}$ ) and with $\mathrm{C}$ doping (Wafer C) in the buffer.

the GaN buffer. Many other works show breakdown data for $\mathrm{C}$ doped devices only and/or for ungated ohmic-to-ohmic isolation structures.

The purpose of this work is to provide physical insight into the off-state, three-terminal, lateral breakdown in AlGaN/GaN HEMTs for power switching applications and to highlight the role of avalanche generation and the other possible breakdown limiting phenomena.

†Corresponding author: nicolo.zagni@unimore.it

Tel: +39-(059)-205-6320

Published version DOI: 10.1016/j.microrel.2019.06.066. (C) 2019, Elsevier. This manuscript version is made available under the CC-BY-NC-ND 4.0 license http://creativecommons.org/licenses/by-nc-nd/4.0/ 


\section{Device structures and simulation models}

In this work, device structures and experimental data from [1] have been adopted as a reference for the numerical simulations carried out with the aim of clarifying the possible physical mechanisms limiting

Tab. I. Geometrical and model parameters of the undoped (Wafer A) device used in the simulations.

\begin{tabular}{lrlr}
\hline \multicolumn{1}{c}{ Geometrical Parameters $(\boldsymbol{\mu m})$} & \multicolumn{2}{c}{ Model Parameters } \\
\hline GaN:UID Channel Thickness & 1.65 & Ir/Ti/Au Gate Schottky Barrier $(\mathrm{eV})$ & 1.0 \\
AlGaN Barrier Thickness & 0.025 & TiN S/D Workfunction $(\mathrm{eV})$ & 4.1 \\
$\mathrm{Si}_{3} \mathrm{~N}_{4}$ Passivation Thickness & 0.15 & $\mathrm{~S} / \mathrm{D}$ Contact Resistance $(\Omega . \mathrm{mm})$ & 0.2 \\
S/D Contact Length & 0.1 & Low-field mobility $\mu_{\mathrm{n}}\left(\mathrm{cm}^{2} / \mathrm{V} . \mathrm{s}\right)$ & 1800 \\
Gate-to-Source Length $L_{\mathrm{GS}}$ & 1 & Saturation velocity $\mathrm{v}_{\mathrm{sat}}(\mathrm{cm} / \mathrm{s})$ & $1.5 \times 10^{7}$ \\
Gate-to-Drain Length $L_{\mathrm{GD}}$ & $1-15$ & UID Doping $\left(\mathrm{cm}^{-3}\right)$ & $1 \times 10^{15}$ \\
Gate Extension (Source) Length & 0.2 & Deep Acceptor Traps Conc. $\mathrm{N}_{\mathrm{Al}}\left(\mathrm{cm}^{-3}\right)$ & $5 \times 10^{15}$ \\
Gate Length $L_{\mathrm{G}}$ & 0.7 & Deep Acceptor Traps Level $E_{\mathrm{A} 1}-E_{\mathrm{V}}(\mathrm{eV})$ & 0.6 \\
Gate Extension (Drain) Length $L_{\mathrm{FP}}$ & 0.6 & Deep Donor Traps Conc. $\mathrm{N}_{\mathrm{D} 1}\left(\mathrm{~cm}^{-3}\right)$ & $1 \times 10^{16}$ \\
& & Deep Donor Traps Level $E_{\mathrm{C}}-E_{\mathrm{D} 1}(\mathrm{eV})$ & 1.0 \\
& & Al molar fraction x $\left(\mathrm{Al}_{\mathrm{x}} \mathrm{Ga}_{1-\mathrm{x}} \mathrm{N}\right)$ & 0.26 \\
& & Polarization Activation & $50 \%$ \\
\hline
\end{tabular}

Tab. II. Geometrical and model parameters of the C-doped (Wafer C) device used in the simulations.

\begin{tabular}{lrlr}
\multicolumn{3}{c}{ Geometrical Parameters $(\boldsymbol{\mu m})$} & \multicolumn{2}{c}{ Model Parameters } \\
\hline GaN:UID Buffer Thickness & 0.250 & Ir/Ti/Au Gate Schottky Barrier $(\mathrm{eV})$ & 1.0 \\
GaN:C Back Barrier Thickness & 1.5 & TiN S/D Workfunction $(\mathrm{eV})$ & 4.1 \\
GaN:UID Channel Thickness & 0.035 & S/D Contact Resistance $(\Omega . m m)$ & 0.2 \\
AlGaN Barrier Thickness & 0.025 & Low-field mobility $\mu_{\mathrm{n}}\left(\mathrm{cm}^{2} / \mathrm{V} . \mathrm{s}\right)$ & 1800 \\
Si $_{3} \mathrm{~N}_{4}$ Passivation Thickness & 0.15 & Saturation velocity $\mathrm{v}_{\mathrm{sat}}(\mathrm{cm} / \mathrm{s})$ & $1.5 \times 10^{7}$ \\
S/D Contact Length & 0.1 & UID Doping $\left(\mathrm{cm}^{-3}\right)$ & $1 \times 10^{15}$ \\
Gate-to-Source Length $L_{\mathrm{GS}}$ & 1 & Deep Acceptor Conc. $N_{\mathrm{DA}}\left(\mathrm{cm}^{-3}\right)$ & $8 \times 10^{17}$ \\
Gate-to-Drain Length $L_{\mathrm{GD}}$ & $1-5$ & Deep Acceptor Level $E_{\mathrm{DA}}-E_{\mathrm{V}}(\mathrm{eV})$ & 0.9 \\
Gate Extension (Source) Length & 0.2 & Deep Donor Conc. $N_{\mathrm{DD}}\left(\mathrm{cm}^{-3}\right)$ & $4 \times 10^{17}$ \\
Gate Length $L_{\mathrm{G}}$ & 0.7 & Deep Donor Level $E_{\mathrm{C}}-E_{\mathrm{DD}}(\mathrm{eV})$ & 0.11 \\
Gate Extension (Drain) Length $L_{\mathrm{FP}}$ & 0.6 & Al molar fraction x $\left(\mathrm{Al}_{\mathrm{x}} \mathrm{Ga}_{1-\mathrm{x}} \mathrm{N}\right)$ & 0.25 \\
& & Polarization Activation & $50 \%$ \\
\end{tabular}



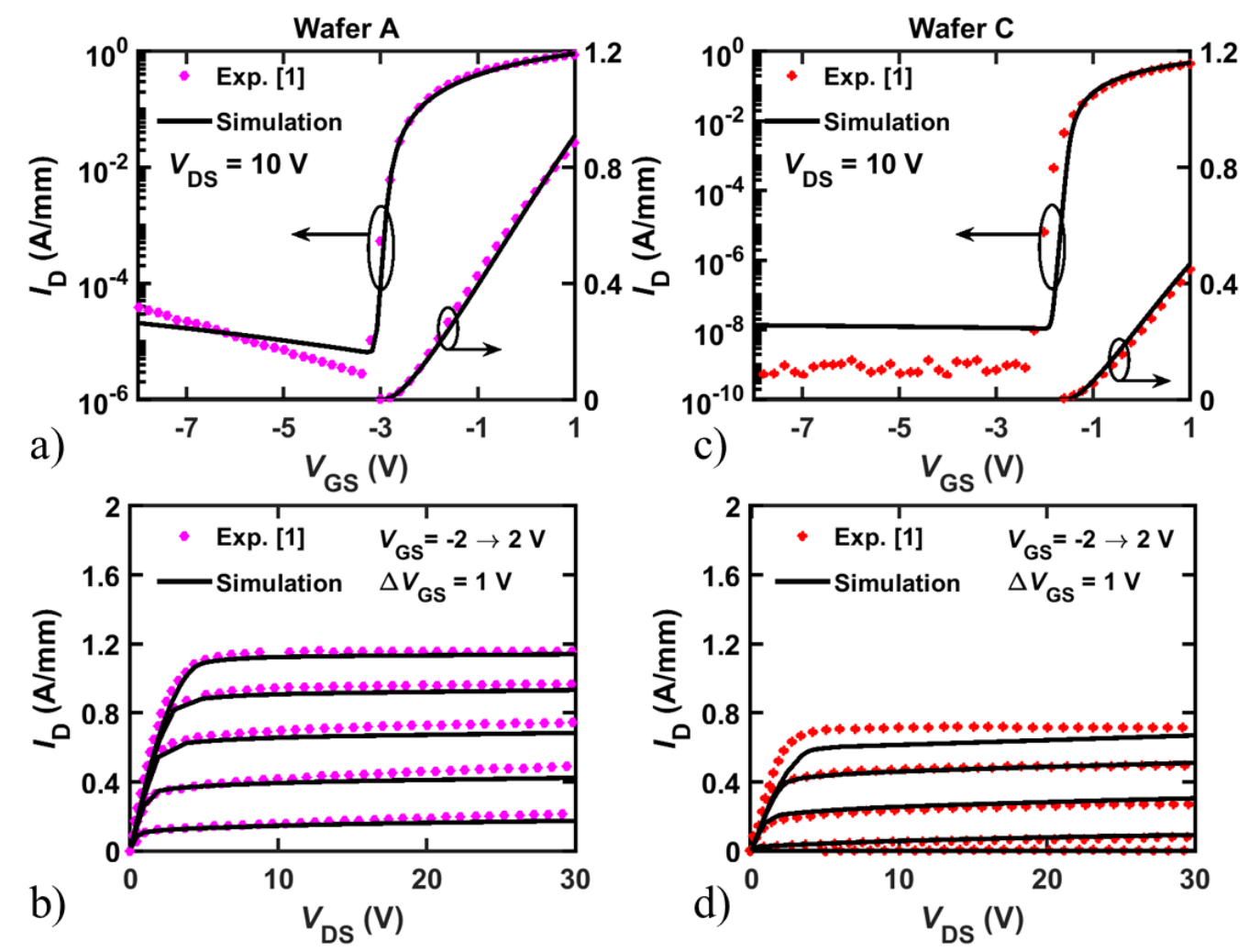

Fig. 2. $I_{\mathrm{D}}-V_{\mathrm{GS}}$ (transfer) and $I_{\mathrm{D}}-V_{\mathrm{DS}}$ (output) curves for a, b) Wafer A and c, d) Wafer C. For the transfer characteristic, $V_{\mathrm{DS}}$ is set to $10 \mathrm{~V}$. For the output characteristic, $V_{\mathrm{GS}}$ is swept between -2 and $2 \mathrm{~V}$ at $1 \mathrm{~V}$ steps. Good overall agreement was found between experimental data [1] (symbols) and simulations (lines).

Tab. III. Chynoweth's Law coefficients values for GaN and AlGaN for the Wafer A and Wafer C devices. Values are taken from Monte Carlo simulations reported in [4] .

\begin{tabular}{|c|c|c|c|c|c|c|}
\hline \multicolumn{7}{|c|}{ Impact Ionization - Chynoweth's Law Coefficients } \\
\hline & \multicolumn{2}{|l|}{ GaN } & \multicolumn{2}{|c|}{$\begin{array}{l}\mathrm{Al}_{\mathrm{x}} \mathrm{Ga} 1-\mathrm{x} N(\mathrm{x}=0.26) \\
\text { Wafer A }\end{array}$} & \multicolumn{2}{|c|}{$\begin{array}{l}\operatorname{Al}_{x} G a 1-x N(x=0.25) \\
\text { Wafer C }\end{array}$} \\
\hline & Electrons & Holes & Electrons & Holes & Electrons & Holes \\
\hline $\mathrm{a}\left(\mathrm{cm}^{-1}\right)$ & $2.32 \times 10^{6}$ & $5.41 \times 10^{6}$ & $3.76 \times 10^{6}$ & $4.76 \times 10^{6}$ & $3.94 \times 10^{6}$ & $4.89 \times 10^{6}$ \\
\hline $\mathrm{b}(\mathrm{V} / \mathrm{cm})$ & $1.4 \times 10^{7}$ & $1.89 \times 10^{7}$ & $2.71 \times 10^{7}$ & $2.79 \times 10^{7}$ & $2.71 \times 10^{7}$ & $2.79 \times 10^{7}$ \\
\hline
\end{tabular}

$V_{\mathrm{BR}}$. A sketch of the analyzed device structures is shown in Fig. 1. Gate-source spacing $\left(L_{\mathrm{GS}}\right)$, gate length $\left(L_{G}\right)$ and gate field plate overhang $\left(L_{\mathrm{FP}}\right)$ are for all structures 1, 0.7, $0.6 \mu \mathrm{m}$, respectively. More details on device fabrication can be found in [1]. The substrate is semi-insulating $\mathrm{SiC}$. The substrate contact was left floating during both measurements [1] and simulations, so that vertical breakdown is not expected to play a role for the voltage range and gateto-drain spacing under investigation in this work. Device simulations were carried out by means of the
Sentaurus Device simulator (Synopsys). Carrier distribution was modeled with Fermi-Dirac statistic, SRH recombination was included as well as mobility degradation due to doping and high field. Piezoelectric polarization was included by using the strain model included in the simulator. Both gate and source/drain contacts were modeled as Schottky contacts with proper barrier/workfunction. Electron tunneling was activated at the contacts to properly reproduce leakage currents (at the gate) and to mimic ohmic contacts (at the source and drain) [3]. In the 
simulations, all process and geometrical parameters were set to their nominal values stated in [1]. A list of the parameters used in the simulation is reported in Tab. I and Tab. II for the undoped and C-doped devices, respectively. Impact-ionization coefficients for both electrons and holes were set in agreement with recent Monte-Carlo calculations [4]. Parameters used in the Cynoweth's law are listed in Tab. III.

In undoped HEMTs, the GaN buffer was modeled by assuming a pair of intrinsic donor-acceptor traps like in [5]. In C-doped HEMTs, C doping was instead modeled by dominant deep acceptor levels (at $0.9 \mathrm{eV}$ from the valence-band edge, $E_{\mathrm{V}}$ ) partially compensated by shallow donors (close to the conduction-band edge, $E_{\mathrm{C}}$ ). In this way, the effective acceptor trap concentration was only a small fraction of the nominal $\mathrm{C}$ doping density, about $1 \%$. By adjusting the donor/acceptor auto-compensation ratio, this model for $\mathrm{C}$ doping indeed allowed us to accurately reproduce dynamic effects in different power GaN technologies [6]-[8]. As a matter of fact, a higher donor concentration in $\mathrm{GaN}: \mathrm{C}$ compared with the donor density measured in unintentionally doped samples has been confirmed experimentally in [9] and attributed to auto-compensation between Crelated donors and acceptors. Moreover, a similar C model with high donor/acceptor auto-compensation ratio has recently been shown by other authors to be instrumental to achieving a realistic description of breakdown effects in C-doped GaN HEMTs [10].

\section{Results}

Simulations were first calibrated against experimental transfer and output IV curves for both undoped and C-doped devices. The outcomes are illustrated in Fig. 2, showing the experimental and simulated transfer and output characteristics for undoped (Wafer A) and C-doped (Wafer C) devices. As can be noted, a satisfactory agreement was achieved in all cases.

Simulations were then used to analyze the $V_{\mathrm{BR}} \mathrm{vS}$ $L_{\mathrm{GD}}$ scaling. Results are reported in Figs. 3 and 4 , showing the experimental and simulated off-state $I_{\mathrm{D}^{-}}$ $V_{\text {DS }}$ curves for undoped (Wafer A) and C-doped (Wafer C) devices with different $L_{\mathrm{GD}}$ values and the corresponding $V_{\mathrm{BR}}$ vs $L_{\mathrm{GD}}$ plots, respectively. Consistently with [1], $V_{\mathrm{BR}}$ is defined as the $V_{\mathrm{DS}}$ value for which $I_{\mathrm{D}}$ reaches $1 \mathrm{~mA} / \mathrm{mm}$. As can be noted, an overall reasonable agreement is achieved between simulated and experimental data. In particular, the simulations are able to fully capture the completely different behavior exhibited by undoped and C-doped devices in terms of $V_{\mathrm{BR}}$ vs $L_{\mathrm{GD}}$ relationship. Namely,

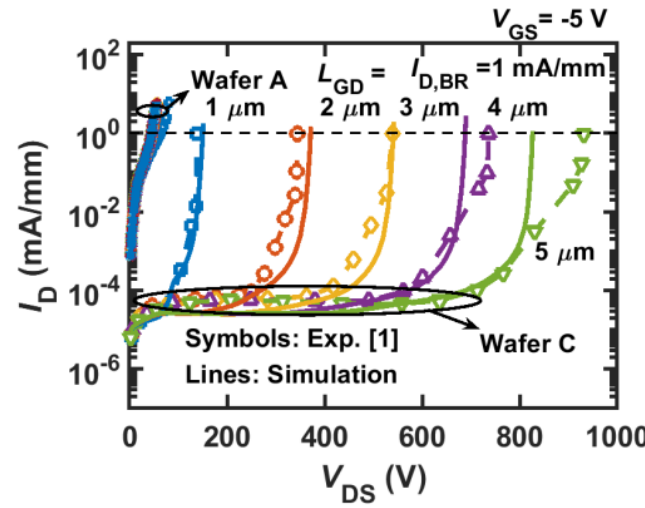

Fig. 3. Experimental [1] (symbols) and simulated (lines) off-state $I_{\mathrm{D}}-V_{\mathrm{DS}}$ curves for undoped (Wafer A) and C-doped (Wafer C) devices with different $L_{\mathrm{GD}}$ values.

$V_{\mathrm{BR}}$ shows no appreciable dependence on $L_{\mathrm{GD}}$ in the HEMT with undoped buffer, whereas it scales almost linearly with $L_{\mathrm{GD}}$ in the HEMT with C-doped buffer. In this latter case, our simulations are in very good agreement with measurements for $L_{\mathrm{GD}}$ ranging from 1 to $3 \mu \mathrm{m}$, while they tend to underestimate $V_{\mathrm{BR}}$ for longer $L_{\mathrm{GD}}$ values, along with a steeper slope than experimental results. The maximum discrepancy (for $L_{\mathrm{GD}}=5 \mu \mathrm{m}$ ) in terms of breakdown voltage matching is however less than $15 \%$ of the experimental value. Simulation results for the C-doped devices were not compared with experiments for $L_{\mathrm{GD}}>6 \mu \mathrm{m}$ because measurements were limited to $V_{\mathrm{DS}}=1000 \mathrm{~V}$ [1], and no breakdown occurred in this range for the longer devices.

We mention the fact that breakdown voltage benefits related to $\mathrm{C}$-doping are expected to come at the price of increased current collapse effects (i.e., degraded $R_{\mathrm{ON}}$ as well as dispersion in the $I_{\mathrm{D}}-V_{\mathrm{DS}}$ as a consequence of increased trapping). Although we did not focus on these aspects in this contribution, it is worth noting that this trade-off is experimentally investigated in the reference paper adopted for calibration of our simulations [1].

Figure 5 shows the simulated $I_{\mathrm{D}}$ up to breakdown for the undoped and C doped devices with $L_{\mathrm{GD}}=2 \mu \mathrm{m}$, along with the corresponding electron currents entering the device from the gate and the source. In both devices, $I_{\mathrm{D}}$ is about equal to the gate electron current up to breakdown, while the source electron current becomes comparable with, and, in the case of the undoped device, even larger than $I_{\mathrm{G}}$ in the breakdown regime. In the device with $\mathrm{C}$-doped buffer, however, $\mathrm{C}$ doping effectively suppresses both gate electron injection and source-drain punch-through, so that breakdown occurs at much higher $V_{\mathrm{DS}}$ (at the same $\left.L_{\mathrm{GD}}\right)$. 


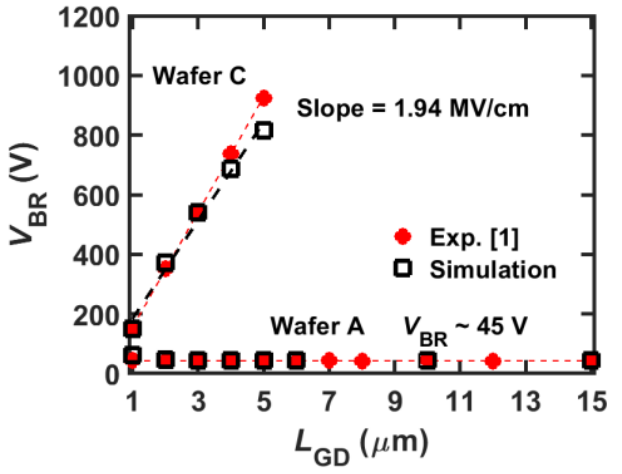

Fig. 4. Experimental [1] (red dots) and simulated (hollow black squares) off-state breakdown voltage $\left(V_{\mathrm{BR}}\right)$ as a function of gate-drain spacing $\left(L_{\mathrm{GD}}\right)$.

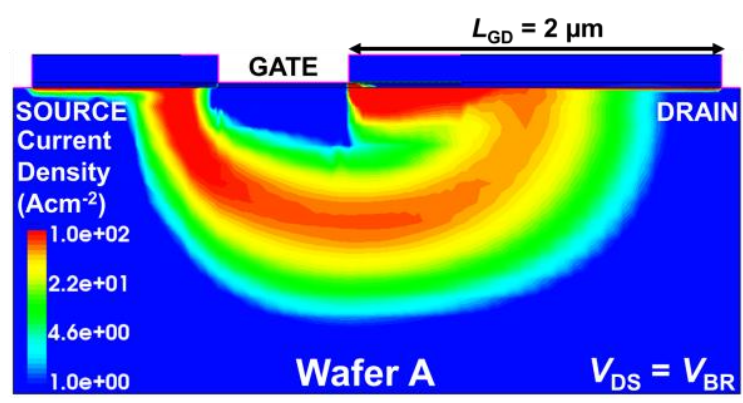

Fig. 6. Contour plot of the electron current density distribution at $V_{\mathrm{DS}}=V_{\mathrm{BR}}$ in the undoped device with $L_{\mathrm{GD}}=2$ $\mu \mathrm{m}$.

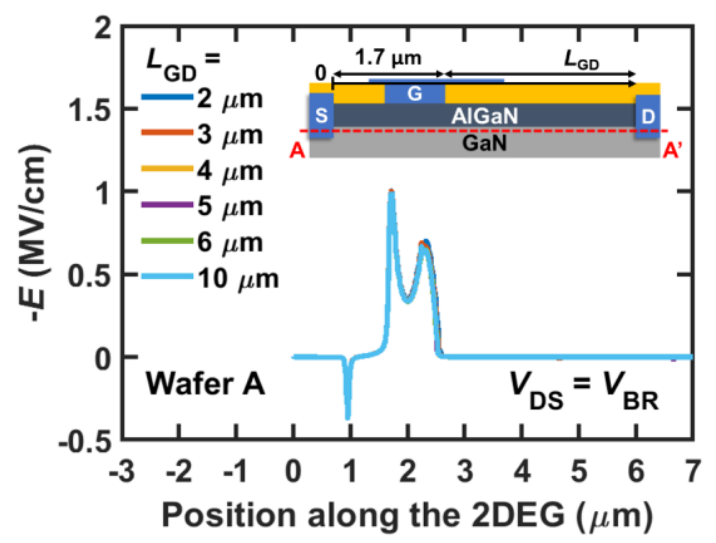

Fig. 8. Parallel component of the electric-field as a function of position along the $\mathrm{AlGaN} / \mathrm{GaN}$ interface in the undoped device for different $L_{\mathrm{GD}}$ values at $V_{\mathrm{DS}}=V_{\mathrm{BR}}$.

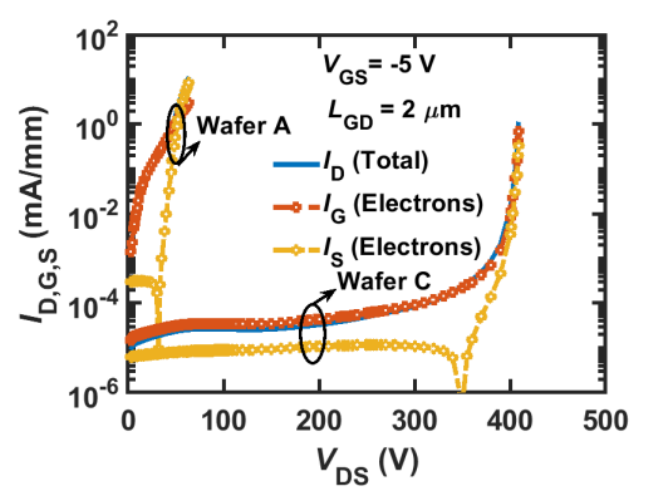

Fig. 5. Simulated drain (blue lines), gate electron (red lines), and source electron (yellow lines) currents as a function of $V_{\mathrm{DS}}$ for the undoped (Wafer $\mathrm{A}$ ) and C-doped (Wafer C) devices having $L_{\mathrm{GD}}=2 \mu \mathrm{m}$.

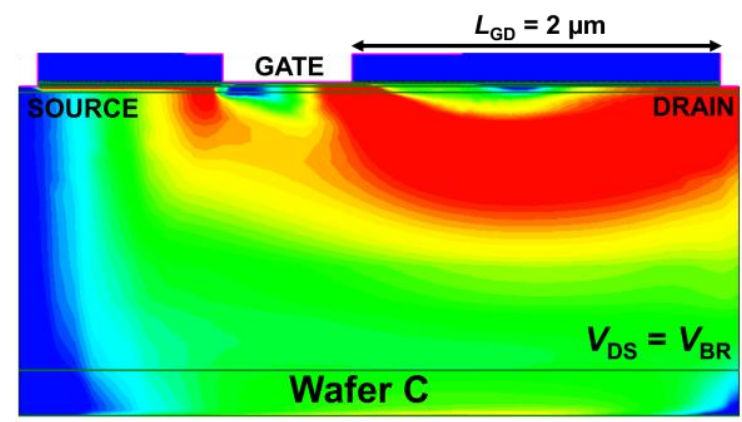

Fig. 7. Contour plot of the electron current density distribution at $V_{\mathrm{DS}}=V_{\mathrm{BR}}$ in the C-doped device with $L_{\mathrm{GD}}=2$ $\mu \mathrm{m}$ (same scale of Fig. 6 is used).

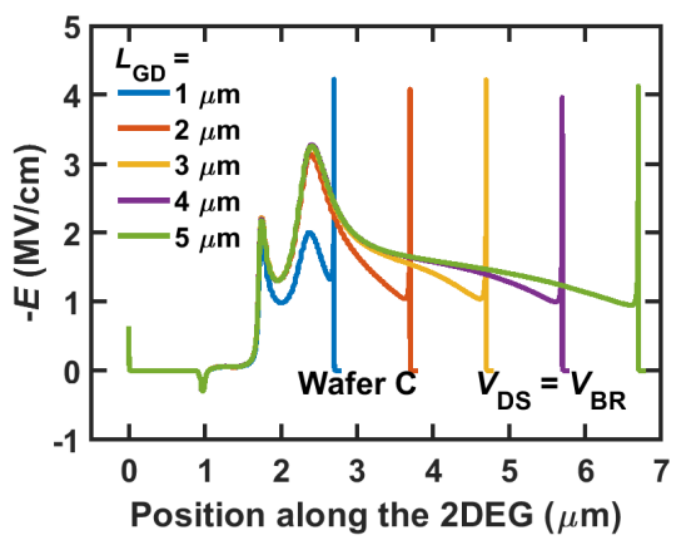

Fig. 9. Parallel component of the electric-field as a function of position along the $\mathrm{AlGaN} / \mathrm{GaN}$ interface in the C-doped device for different $L_{\mathrm{GD}}$ values at $V_{\mathrm{DS}}=V_{\mathrm{BR}}$. 


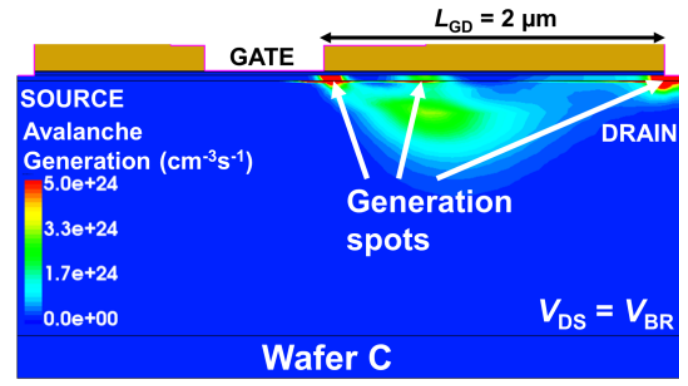

Fig. 10. Contour plot of the avalanche generation distribution at $V_{\mathrm{DS}}=V_{\mathrm{BR}}$ in the C-doped device with $L_{\mathrm{GD}}=2 \mu \mathrm{m}$.

The presence of two conductive paths within the $\mathrm{GaN}$ channel/buffer, one connecting the gate to the drain, the other connecting the source to the drain, is clearly visible in Figs. 6 and 7, showing the 2D electron current density distribution at breakdown in the undoped and the C-doped device with $L_{\mathrm{GD}}=2 \mu \mathrm{m}$, respectively.

Figure 8 shows the electric-field distribution at breakdown (i.e., at the $V_{\mathrm{DS}}$ for which $I_{\mathrm{D}}=1 \mathrm{~mA} / \mathrm{mm}$, consistently with measurements [1]) along the $\mathrm{AlGaN}-\mathrm{GaN}$ interface in the device with undoped buffer for different $L_{\mathrm{GD}}$ values. In this device, the breakdown drain current limit of $1 \mathrm{~mA} / \mathrm{mm}$ is reached due to the combination of gate-injected electron and source-drain punch-through currents. The electric field peak occurs at the drain-end of the gate. Its value is smaller than the critical field for avalanche and is negligibly impacted by changing $L_{\mathrm{GD}}$. In the region between the end of the field plate and the drain contact, the electric field is negligibly small. For these reasons, $V_{\mathrm{BR}}$ is almost insensitive to $L_{\mathrm{GD}}$ as seen in Figs. 3 and 4.

Figure 9 shows the electric-field distribution at breakdown along the AlGaN-GaN interface in the device with C-doped buffer for different $L_{\mathrm{GD}}$ values. In this device, the high field region is effectively distributed throughout the gate-drain access region, and the field is nonnegligible even in the region between the field- plate end and the drain contact. For this reason, increasing $L_{\mathrm{GD}}$ shifts the breakdown to larger voltages. However, the electric field distribution is nonuniform, with distinct peaks at three positions along the $\mathrm{AlGaN}-\mathrm{GaN}$ interface, namely in correspondence of the drain end of the gate, the fieldplate end, and the drain contact. As a result, the $\Delta V_{\mathrm{BR}} / \Delta L_{\mathrm{GD}}$ slope is smaller than $E_{\mathrm{CRIT}}$, even if breakdown is induced by avalanche generation. The critical field for breakdown is specifically reached at the drain contact for all $L_{\mathrm{GD}}$ values considered.

Figure 10 shows the avalanche generation distribution at $V_{\mathrm{DS}}=V_{\mathrm{BR}}$ in the C-doped device with $L_{\mathrm{GD}}=2 \mu \mathrm{m}$, confirming the presence of the three generation spots.

\section{Conclusions}

We have analysed the off-state, three-terminal, lateral breakdown of AlGaN/GaN HEMTs for power switching applications, by comparing twodimensional numerical device simulations with experimental data from device structures with different $L_{\mathrm{GD}}$ and with either undoped or Carbondoped GaN buffer layers. In undoped HEMTs, the breakdown voltage is insensitive to $L_{\mathrm{GD}}$, while it increases linearly with this parameter with $\mathrm{a} \approx 2 \times 10^{6}$ $\mathrm{V} / \mathrm{cm}$ slope in C-doped devices. These aspects are successfully captured by our simulations and are attributed to the different breakdown mechanisms in the two devices, namely: $i)$ a combination of gate electron injection and source-drain punch-through current in undoped HEMTs; and ii) avalanche breakdown triggered by gate electron injection in $\mathrm{C}$ doped HEMTs. In the latter case, the critical field for avalanche is reached at breakdown in correspondence of the drain contact for all $L_{\mathrm{GD}}$ values. The fact that the $\Delta V_{\mathrm{BR}} / \Delta L_{\mathrm{GD}}$ slope is, in spite of this, smaller than the critical field for avalanche is explained by the simulations as a result of the highly nonuniform electric field distribution within the gate-drain access region. Our TCAD model can be useful for designers to predict the voltage handling capabilities of $\mathrm{GaN}$ HEMTs during the device optimization loop, depending on the buffer doping employed. Moreover, it can also be adopted as an aid in the interpretation of failure modes during robustness and off-state stepstress tests.

\section{References}

[1] E. Bahat-Treidel et al., IEEE Trans. Electron Devices, vol. 57, no. 11, pp. 3050-3058, 2010.

[2] A. J. Fischer et al., Electron. Lett., vol. 52, no. 13, pp. 1170-1171, 2016.

[3] V. Joshi et al., IEEE Trans. Electron Devices, vol. 15, no. 6, pp. 947-955, Nov. 2016.

[4] E. Bellotti et al., Journal of Applied Physics 111, 103711 (2012).

[5] M. J. Uren et al., IEEE Trans. Electron Devices, vol. 59, no. 12 , pp. 3327-3333, 2012.

[6] G. Meneghesso et al., Proc. of the IEEE Int. Reliab. Phys. Symp., pp. 6-10, 2014.

[7] G. Verzellesi et al., IEEE Electr. Dev. Lett., vol. 35, no. 4, pp. 443-445, 2014.

[8] A. Chini et al, IEEE Trans. on Electr. Dev., vol. 63, no 9, pp. 3473-3478, 2016

[9] A. Fariza et al., APL, 109, 212102, 2016.

[10] V. Joshi et al., IEEE Trans. on Electr. Dev., vol. 66, no. 1 , pp. 561-569, 2019 